\title{
On the theory of current states in superconducting junctions of SNINS type
}

\author{
V.E.Sakhnyuk, A.V.Svidzynskyj \\ Department of Theoretical and Mathematical Physics, \\ Lesya Ukrainka Volyn States University, \\ 13 Voli Avenue, 43000 Lutsk, Ukraine
}

Received August 3, 2005, in final form November 8, 2005

\begin{abstract}
The behavior of the order parameter close to the NS interface in an SNINS junction is considered. To this end, a linear integral equation, which is valid near the superconductor-normal metal interface, is obtained and researched. The Ginzburg-Landau equation is solved taking into account the effect of the current on the space behaviour of the order parameter. Applying the method of quasiorthogonality to asymptotics, the boundary condition for the Ginzburg-Landau equation is obtained. The calculation of the current states, which can exist in an SNINS junction is carried out. We assume the normal layer thickness to be arbitrary and the temperature to be close to critical.
\end{abstract}

Key words: current, critical temperature, Ginzburg-Landau equation, the order parameter

PACS: $74.50 .+r$

\section{Introduction}

To calculate the current in the superconducting junctions of SNS, SINS, SNINS type (where $S$ - superconductor, $N$ - normal metal, $I$ - insulator) at the temperature not too close to critical, the model with the piecewise constant order parameter $\Delta(\vec{r})$ has been used. In this model a modulus of the order parameter is assumed to be a constant in the superconducting region and zero in the normal metal. Such an approach for the investigation of the current states in pure $S N S$ junction was realized in paper [1]. The calculation of the current taking into account the reflection of electrons on the junction (junctions of SINS, SINIS, SNINS type) has been made in [3-5]. The numerous results in the model with the piecewise constant order parameter are contained in [6].

However, for the temperature close to critical such a reduced model cannot be used. In this region of temperatures the spatial nonhomogeneity of the order parameter near NS interface should be taken into account. In order to investigate the spatial behaviour of the order parameter at a temperature close to critical the Ginzburg-Landau equation has been used. However, the boundary condition has to be taken into account here. One can find the boundary condition from the linear integral equation which is valid near the interface for the distances of the order $\xi_{0}\left(\xi_{0}\right.$ - the length coherence). Taking into account the spatial behaviour of the order parameter the current in $S N S, S I N S$ junctions has been calculated in papers [3,9], while in [7] the $S N I N S$ junction is considered. However, in these papers we have investigated the asymptotic case, when the thickness of the normal layer $d \gg \xi_{0}$.

The purpose of this paper is to investigate the current states in SNINS junction for arbitrary thickness of the normal layer at the temperature close to critical.

*E-mail: sve@lab.univer.lutsk.ua 


\section{The common scheme for the investigation of the behavior of the order parameter near $N S$ interface.}

It is well-known that the information about the spacial behavior of the order parameter can be found from the self-consistent condition $[3,8]$ :

$$
\Delta(\vec{r})=|g| T \sum_{\omega_{n}} F_{\omega_{n}}^{*}(\vec{r}, \vec{r})
$$

where $g$ is the coupling constant, $T$ is temperature, $\omega_{n}=\pi T(2 n+1)$ is the odd Matsubara frequency, $F_{\omega_{n}}(\vec{r}, \vec{r})$ is the so-called anomalous Matsubara Green's function. Both the anomalous Matsubara Green's function and the usual Matsubara Green's function $G_{\omega_{n}}\left(\vec{r}, \overrightarrow{r^{\prime}}\right)$ satisfy the Gorkov's system of equations:

$$
\left\{\begin{array}{l}
\left(\mathrm{i} \omega_{n}-\hat{\xi}\right) G_{\omega_{n}}\left(\vec{r}, \overrightarrow{r^{\prime}}\right)+\Delta(\vec{r}) F_{\omega_{n}}\left(\vec{r}, \overrightarrow{r^{\prime}}\right)=\delta\left(\vec{r}-\overrightarrow{r^{\prime}}\right) \\
\left(\mathrm{i} \omega_{n}+\hat{\xi}\right) F_{\omega_{n}}\left(\vec{r}, \overrightarrow{r^{\prime}}\right)+\stackrel{\Delta}{\Delta}(\vec{r}) G_{\omega_{n}}\left(\vec{r}, \overrightarrow{r^{\prime}}\right)=0
\end{array}\right.
$$

Here $\hat{\xi}=\hat{\vec{p}}^{2} /(2 m)-\mu+V(\vec{r})$, where $V(\vec{r})$ is the potential, which we will later take as $\delta$ - functional. Let us suppose that the magnetic field is absent. Thus, in order to obtain the equation for $\Delta(\vec{r})$, it is necessary to express the function $F_{\omega_{n}}\left(\vec{r}, \vec{r}^{\prime}\right)$ from system $(2)$ and substitute it into the selfconsistent condition (1). In the general case this problem is very complicated. However, for the temperature close to critical the order parameter $\Delta(\vec{r})$ is small, so that Green's functions can be expanded into a series in terms of the $\Delta(\vec{r})$. For $F_{\omega_{n}}\left(\vec{r}, \vec{r}^{\prime}\right)$ in the linear approximation one has

$$
F_{\omega_{n}}(\vec{r}, \vec{r})=\int G_{-\omega_{n}}^{(0)}\left(\vec{r}, \vec{r}^{\prime}\right) G_{\omega_{n}}^{(0)}\left(\vec{r}^{\prime}, \vec{r}\right) \Delta^{*}\left(\vec{r}^{\prime}\right) \mathrm{d} \vec{r}^{\prime}
$$

Inserting (3) into (1) we obtain the linear integral equation

$$
\Delta(\vec{r})=\int K\left(\vec{r}, \vec{r}^{\prime}\right) \Delta\left(\vec{r}^{\prime}\right) \mathrm{d} \vec{r}^{\prime}
$$

with the kernel

$$
K\left(\vec{r}, \vec{r}^{\prime}\right)=|g| T_{\mathrm{c}} \sum_{\omega_{n}} G_{-\omega_{n}}^{(0)}\left(\vec{r}, \vec{r}^{\prime}\right) G_{\omega_{n}}^{(0)}\left(\vec{r}^{\prime}, \vec{r}\right),
$$

where $G_{\omega_{n}}^{(0)}\left(\vec{r}, \vec{r}^{\prime}\right)$ is the Green's function of the structure under investigation in the normal states. It satisfies the equation

$$
\left(\mathrm{i} \omega_{n}-\hat{\xi}\right) G_{\omega_{n}}^{(0)}\left(\vec{r}, \vec{r}^{\prime}\right)=\delta\left(\vec{r}^{\prime}-\vec{r}\right) .
$$

In order to obtain the explicit form of the linear integral equation (4) describing the behaviour of the order parameter near $N S$-interface in a $S N I N S$ junction, it is necessary to have the expression for the Green's function $G_{\omega_{n}}^{(0)}\left(\vec{r}, \vec{r}^{\prime}\right)$. This function can be found from equation (5) in which $\hat{\xi}=$ $\hat{\vec{p}}^{2} /(2 m)-\mu+U_{0} \delta(z)$, where the last term simulates the thin layer of an insulator placed in the middle of the normal region (plane $X 0 Y$ ). The best way of constructing a Green's function is to solve the corresponding one-particle problem (see e.g. [10]). After substituting the expressions for the Green's function in the formula for $K\left(\vec{r}, \vec{r}^{\prime}\right)$ one can carry out the averaging on the atom distance [2]. Thus, we obtain the linear integral equation for the order parameter

$$
\begin{aligned}
\Delta(\zeta)= & \frac{\rho}{2} \sum_{n} \int_{-\infty}^{-d / 2} \mathrm{~d} \zeta^{\prime} \Delta\left(\zeta^{\prime}\right) \int_{0}^{1} \frac{\mathrm{d} x}{x} \exp \left(-\frac{|2 n+1|}{x}\left|\zeta-\zeta^{\prime}\right|\right) \\
& +\frac{\rho}{2} \sum_{n} \int_{d / 2}^{\infty} \mathrm{d} \zeta^{\prime} \Delta\left(\zeta^{\prime}\right) \int_{0}^{1} \frac{\mathrm{d} x}{x} \operatorname{sign} \zeta \zeta^{\prime} R(x) \exp \left(-\frac{|2 n+1|}{x}\left(|\zeta|+\left|\zeta^{\prime}\right|\right)\right) .
\end{aligned}
$$


Here we take into account that the spatial homogeneity is broken only in the direction of $0 Z$ axis. Therefore, we introduced the dimensionless quantities: $\rho=|g| N(0)$ is dimensionless coupling constant, $N(0)$ is electron state density on the surface of the Fermisphere; $\zeta=z / \xi_{0}$ is dimensionless variable; $R(x)$ is coefficient of the reflection, $a=d / \xi_{0}$ is dimensionless thickness of the normal layer. Hereinafter, it is convenient to consider the equation (6) separately in the regions $\zeta>a / 2$ and $\zeta<-a / 2$, i. e. in both the right superconductor and the left one. Then, introducing the even $\Delta_{\mathrm{S}}(\zeta)=1 / 2(\Delta(\zeta)+\Delta(-\zeta))$, and the odd $\Delta_{a}(\zeta)=1 / 2(\Delta(\zeta)-\Delta(-\zeta))$ parts of the order parameter $\Delta(\zeta)$, one can obtain the following equations

$$
\begin{aligned}
& \Delta_{\mathrm{s}}(\zeta)=\int_{0}^{\infty} \mathrm{d} \zeta^{\prime} \Delta_{\mathrm{s}}\left(\zeta^{\prime}\right)\left\{K\left(\zeta-\zeta^{\prime}\right)+K\left(\zeta+\zeta^{\prime}+a\right)\right\} \\
& \Delta_{a}(\zeta)=\int_{0}^{\infty} \mathrm{d} \zeta^{\prime} \Delta_{a}\left(\zeta^{\prime}\right)\left\{K\left(\zeta-\zeta^{\prime}\right)+K_{\mathrm{D}}(\zeta+\zeta+a)\right\} .
\end{aligned}
$$

Here the shift of variables was performed: $\zeta \rightarrow \zeta+a / 2, \zeta^{\prime} \rightarrow \zeta^{\prime}+a / 2$ and the shifted functions are denoted by the same letters, i. e. $\Delta_{\mathrm{S}}(\zeta+a / 2) \rightarrow \Delta_{\mathrm{S}}(\zeta), \Delta_{a}(\zeta+a / 2) \rightarrow \Delta_{a}(\zeta)$. Furthermore, the following notations are introduced

$$
\begin{aligned}
K(\zeta) & =\frac{\rho}{2} \sum_{n} \int_{0}^{1} \frac{\mathrm{d} x}{x} \exp \left(-\frac{|2 n+1|}{x}|\zeta|\right), \\
K_{\mathrm{D}}(\zeta) & =\frac{\rho}{2} \sum_{n} \int_{0}^{1} \frac{\mathrm{d} x}{x} \tau(x) \exp \left(-\frac{|2 n+1|}{x}|\zeta|\right),
\end{aligned}
$$

where $\tau(x)=1-2 D(x)$. $D(x)$ is the coefficient of transition across the insulator.

From equations (7) and (8) it follows that the asymptotes of $\Delta_{\mathrm{S}}(\zeta)$ and $\Delta_{a}(\zeta)$ are linear at infinity $(\zeta \rightarrow \infty)$.

Let us substitute the expressions for $\Delta_{\mathrm{S}}(\zeta)$ and $\Delta_{a}(\zeta)$ with separated asymptote

$$
\begin{aligned}
& \Delta_{\mathrm{s}}(\zeta)=C_{1}\left(\zeta+q_{1, \infty}+\psi_{\mathrm{s}}(\zeta)\right), \\
& \Delta_{a}(\zeta)=C_{2}\left(\zeta+q_{2, \infty}+\psi_{a}(\zeta)\right) \text {, } \\
& \zeta \rightarrow \infty, \quad \lim _{\zeta \rightarrow \infty} \psi_{s, a}(\zeta)=0
\end{aligned}
$$

into (7) and (8), respectively.

It is important to calculate the coefficients $q_{1, \infty}$ and $q_{2, \infty}$, since they are included into the expression for the current. To calculate them we used the method of quasiorthogonality to asymptotics [11]. As a result we obtain two systems

$$
\begin{aligned}
& \left\{\begin{array}{l}
\int_{0}^{\infty} \mathrm{d} \zeta \int_{0}^{\infty}\left\{K\left(\zeta+\zeta^{\prime}\right)-K\left(\zeta+\zeta^{\prime}+a\right)\right\} q_{\mathrm{s}}\left(\zeta^{\prime}\right) \mathrm{d} \zeta^{\prime}=I_{1}+I_{1}(a), \\
\int_{0}^{\infty} \mathrm{d} \zeta \zeta \int_{0}^{\infty}\left\{K\left(\zeta+\zeta^{\prime}\right)+K\left(\zeta+\zeta^{\prime}+a\right)\right\} q_{\mathrm{s}}\left(\zeta^{\prime}\right) \mathrm{d} \zeta^{\prime}=2 q_{1, \infty} I_{1}-I_{2}-I_{2}(a),
\end{array}\right. \\
& \left\{\begin{array}{l}
\int_{0}^{\infty} \mathrm{d} \zeta \int_{0}^{\infty}\left\{K\left(\zeta+\zeta^{\prime}\right)-K_{\mathrm{D}}\left(\zeta+\zeta^{\prime}+a\right)\right\} q_{a}\left(\zeta^{\prime}\right) \mathrm{d} \zeta^{\prime}=I_{1}+I_{1}(D, a), \\
\int_{0}^{\infty} \mathrm{d} \zeta \zeta \int_{0}^{\infty}\left\{K\left(\zeta+\zeta^{\prime}\right)+K_{\mathrm{D}}\left(\zeta+\zeta^{\prime}+a\right)\right\} q_{a}\left(\zeta^{\prime}\right) \mathrm{d} \zeta^{\prime}=2 q_{2, \infty} I_{1}-I_{2}-I_{2}(D, a) .
\end{array}\right.
\end{aligned}
$$

Here new functions: $q_{\mathrm{s}}(\zeta)=q_{1, \infty}+\psi_{\mathrm{s}}(\zeta), q_{a}(\zeta)=q_{2, \infty}+\psi_{a}(\zeta)$ are introduced, and the following notation is used:

$$
I_{0}(D, a)=\int_{0}^{\infty} \mathrm{d} \zeta \int_{0}^{\infty} \mathrm{d} \zeta^{\prime} K_{\mathrm{D}}\left(\zeta+\zeta^{\prime}+a\right), \quad I_{1}(D, a)=\int_{0}^{\infty} \mathrm{d} \zeta \int_{0}^{\infty} \mathrm{d} \zeta^{\prime} \zeta^{\prime} K_{\mathrm{D}}\left(\zeta+\zeta^{\prime}+a\right),
$$




$$
\begin{aligned}
I_{2}(D, a) & =\int_{0}^{\infty} \mathrm{d} \zeta \zeta \int_{0}^{\infty} \mathrm{d} \zeta^{\prime} \zeta^{\prime} K_{\mathrm{D}}\left(\zeta+\zeta^{\prime}+a\right), \quad I_{k}(a)=I_{k}(0, a), \quad I_{k}=I_{k}(0,0), \\
k & =0,1,2 .
\end{aligned}
$$

Note also that

$$
I_{0}=\frac{\rho}{4} S_{2}, \quad I_{1}=\frac{\rho}{6} S_{3}, \quad I_{2}=\frac{\rho}{8} S_{4}, \quad S_{\alpha}=\sum_{n=-\infty}^{\infty} \frac{1}{|2 n+1|^{\alpha}} .
$$

Let us take trial functions $\Gamma_{\mathrm{s}}, \Gamma_{a}$ instead of $q_{\mathrm{s}}\left(\zeta^{\prime}\right), q_{a}\left(\zeta^{\prime}\right)$, which are put to be indefinite constants. Then from (10) and (11) one has the final form expressions for $q_{1, \infty}, q_{2, \infty}$

$$
\begin{aligned}
q_{1, \infty} & =\frac{1}{2 I_{1}}\left\{I_{2}+I_{2}(a)+\frac{\left(I_{1}+I_{1}(a)\right)^{2}}{I_{0}-I_{0}(a)}\right\}, \\
q_{2, \infty} & =\frac{1}{2 I_{1}}\left\{I_{2}+I_{2}(D, a)+\frac{\left(I_{1}+I_{1}(D, a)\right)^{2}}{I_{0}-I_{0}(D, a)}\right\} .
\end{aligned}
$$
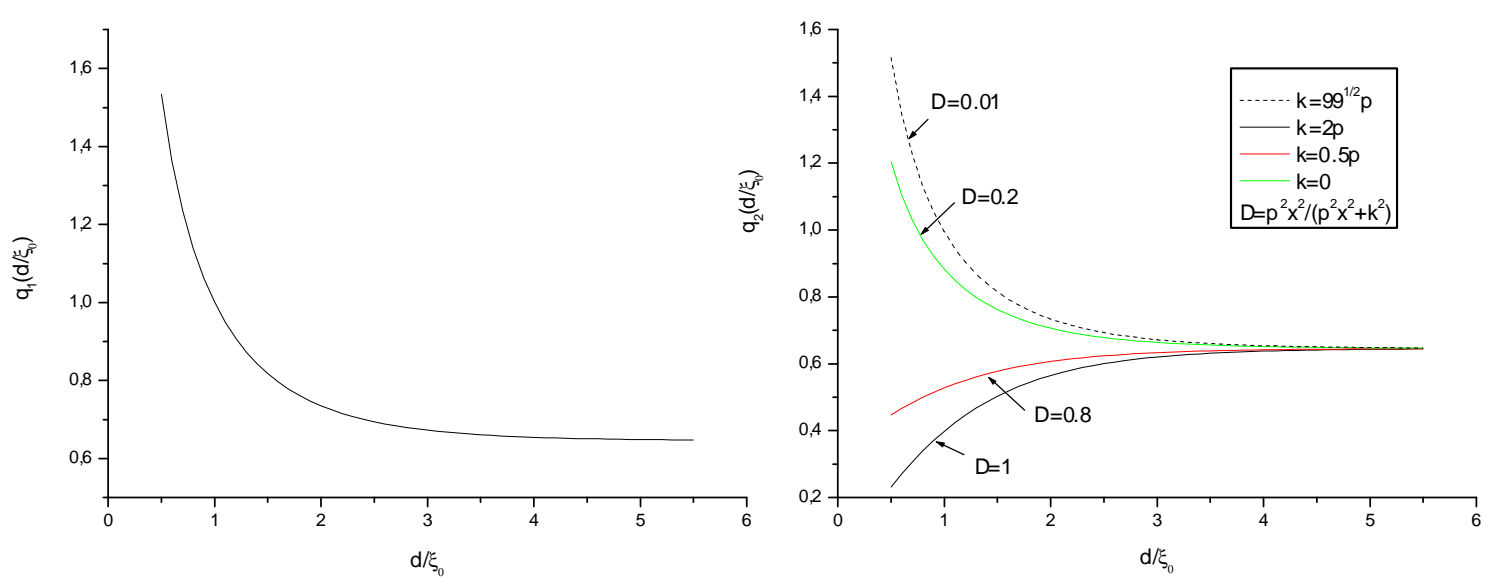

Figure 1. $q_{1}$ and $q_{2}$ as functions on the normal layer thickness $d / \xi_{0}$.

From figure 1 one can see that the difference between $q_{1}$ and $q_{2}$ increases at the small thickness of the normal layer. It is the consequence of a marked increase in coupling of the supercoductors. In the opposite case $q_{1}$ and $q_{2}$ are close to 0.64 , which is characteristic of NS - junction.

Assuming the transition coefficient of electrons $D$ in (14) to be equal to unity, we can reproduce the well-known result for an $S N S$ junction [12].

\section{Solution of the Ginzburg-Landau equation for the order parameter which takes into account a superfluid velocity}

Let the order parameter be small and let us assume that $\Delta(\vec{r})$ is a slowly changing function; such a region must exist, since the order parameter tends to a constant in the depth of a superconductor. Then, it is necessary to continue the expansion (3) to the third power of the order parameter $\Delta(\vec{r})$. However, the last term can be taken in the local form. As a result we come to the nonlinear second order differential equation, which is called the Ginzburg-Landau equation. Let us write down this equation

$$
\frac{\xi^{2}(T)}{\xi_{0}^{2}} \frac{\mathrm{d}^{2} \Delta(\zeta)}{\mathrm{d} \zeta^{2}}-\frac{1}{\Delta_{\infty}^{2}}|\Delta(\zeta)|^{2} \Delta(\zeta)+\Delta(\zeta)=0
$$


where

$$
\Delta_{\infty}^{2}=\frac{8 \pi^{2} T_{\mathrm{c}}^{2}}{7 \zeta(3)}\left(1-\frac{T}{T_{\mathrm{c}}}\right), \quad \xi(T)=\sqrt{\frac{7 \zeta(3)}{12}} \frac{\xi_{0}}{\sqrt{1-T / T_{\mathrm{c}}}}
$$

are the magnitude of both the order parameter at infinity and a characteristic length of the change of the order parameter close to critical temperature, respectively; $\xi_{0}=v_{0} /\left(2 \pi T_{\mathrm{c}}\right)-$ a coherence length, $\zeta=z / \xi_{0}-$ a dimensionless variable.

Since we investigate the junctions at the presence of the current states, the solution of equation (15) has to be presented in the form

$$
\Delta(\zeta)=\mathrm{e}^{ \pm \mathrm{i} \varphi / 2} \Delta_{\infty} f(\zeta) \mathrm{e}^{2 \mathrm{i} m \chi(\zeta)} .
$$

A phase $\chi(\zeta)$ of the order parameter is introduced here. It is bound up with a superfluid velocity: $\nabla \chi=v_{\mathrm{s}}$. This function is considered to be continuous throughout. Specifically, we assume that $\chi(d / 2)=\chi(-d / 2)=0$. To describe the current states we should assume the presence of a phase difference between the banks of the junction. To this end, we introduce the phase factor $\exp \{ \pm \mathrm{i} \varphi / 2\}$, which is independent of the coordinates within each of the superconductors, but differs in both of them. We shall assume that the phase $-\varphi / 2$ is for the left hand superconductor and the phase $\varphi / 2$ is for right hand one. After substituting (16) into (15) and separating both the real part and the imaginary one, we obtain

$$
\begin{gathered}
\frac{\xi^{2}(T)}{\xi_{0}^{2}} f^{\prime \prime}(\zeta)-\xi^{2}(T) 4 m^{2} v_{\mathrm{s}}^{2}(\zeta) f(\zeta)+f(\zeta)-f^{3}(\zeta)=0 \\
v_{\mathrm{s}}(\zeta) f^{2}(\zeta)=\text { const. }
\end{gathered}
$$

At infinity $f$ is a constant and one gets from equation (17)

$$
f_{\infty}^{2}=1-4 m^{2} v_{\mathrm{s}}^{2}(\infty) \xi^{2}(T), \quad f_{\infty} \equiv f(\infty) .
$$

Based on both the last relation and the equation (18) we have

$$
v_{\mathrm{s}}^{2}(\zeta)=\frac{1-f_{\infty}^{2}}{4 m^{2} \xi^{2}(T)} \frac{f_{\infty}^{4}}{f^{4}(\zeta)} .
$$

Substituting (20) into (17) one can obtain the equation for $f(\zeta)$

$$
\frac{\xi^{2}(T)}{\xi_{0}^{2}} f^{\prime \prime}(\zeta)-\left(1-f_{\infty}^{2}\right) \frac{f_{\infty}^{4}}{f^{3}(\zeta)}+f(\zeta)-f^{3}(\zeta)=0 .
$$

The last equation has the first integral

$$
\frac{\xi^{2}(T)}{\xi_{0}^{2}}\left(f^{\prime}(\zeta)\right)^{2}+\left(1-f_{\infty}^{2}\right) f_{\infty}^{2}\left(\frac{f_{\infty}^{2}}{f^{2}(\zeta)}-1\right)+f^{2}(\zeta)-f_{\infty}^{2}+\frac{1}{2} f_{\infty}^{4}-\frac{1}{2} f^{4}(\zeta)=0 .
$$

Finally, carrying out the integration, we obtain

$$
f(\zeta)=\left\{2\left(1-f_{\infty}^{2}\right)+\left(3 f_{\infty}^{2}-2\right) \operatorname{th}^{2} \frac{\zeta+C}{\sqrt{2} \xi(T)} \xi_{0}\right\}^{1 / 2} .
$$

Another form of the solution which includes the superfluid velocity is

$$
f(\zeta)=\left\{1-4 m^{2} v_{\mathrm{s}}^{2}(\infty) \xi^{2}(T)+\frac{12 m^{2} v_{\mathrm{s}}^{2}(\infty) \xi^{2}(T)-1}{\operatorname{ch}^{2} \frac{\zeta+C}{\sqrt{2} \xi(T)} \xi_{0}}\right\}^{1 / 2} .
$$

Comparing the obtained result (formula (22)) with the one which was known before ([8], section 3, equation (4.5)) it is easy to see that taking into account the term with the superfluid velocity in the Ginzburg-Landau equation brings a new component in the expression for $f(\zeta)$. 


\section{Boundary value of the order parameter}

The obtained solution of the Ginzburg-Landau equation contains an unknown constant $C$. In order to fix it, it is necessary to have the boundary condition for the function $f(\zeta)$ at $\zeta=a / 2$.

Let us put $\zeta=a / 2$ in equation (21) then, introducing the notation $f(a / 2) \equiv f_{+}$, one can obtain the relation between $f_{+}^{\prime}$ and $f_{+}$

$$
\frac{\xi(T)}{\xi_{0}} f_{+}^{\prime}-\frac{f_{\infty}^{2}-f_{+}^{2}}{f_{+}} \sqrt{f_{\infty}^{2}-1+\frac{1}{2} f_{+}^{2}}=0
$$

Another relation between $f_{+}^{\prime}$ and $f_{+}$we have from conditions on the NS interface (see [9])

$$
\frac{f_{+}^{\prime}}{f_{+}}=\frac{\cos ^{2} \frac{\varphi}{2}}{q_{1, \infty}}+\frac{\sin ^{2} \frac{\varphi}{2}}{q_{2, \infty}} \equiv \frac{1}{q_{\infty}} .
$$

Using (25) let us exclude $f_{+}^{\prime}$ from (24), as a result we come to the closed equation with respect to $f_{+}$:

$$
f_{+}^{6}-2\left(1+\frac{\xi^{2}(T)}{\xi_{0}^{2} q_{\infty}^{2}}\right) f_{+}^{4}+f_{\infty}^{2}\left(4-3 f_{\infty}^{2}\right) f_{+}^{2}+2 f_{\infty}^{4}\left(f_{\infty}^{2}-1\right)=0
$$

$f_{\infty}$ satisfies such an equation

$$
f_{\infty}^{6}-f_{\infty}^{4}+\left(\frac{\xi(T)}{2 \xi_{0}}\right)^{2}\left(\frac{1}{q_{2, \infty}}-\frac{1}{q_{1, \infty}}\right)^{2} \sin ^{2} \varphi f_{+}^{4}=0
$$

Indeed, using the condition (6.21) in [9] from equation (18) one has

$$
v_{\mathrm{s}}(\infty)=\frac{\sin \varphi}{4 m \xi_{0}}\left(\frac{1}{q_{2, \infty}}-\frac{1}{q_{1, \infty}}\right) \frac{f_{+}^{4}}{f_{\infty}^{2}} .
$$

Then substituting (28) into (19) we come to (27).

Let us return to equation (26). It is a cube equation with respect to $f_{+}^{2} \equiv x$ and if we introduce the following notation:

$$
a=-2\left(1+\frac{\xi^{2}(T)}{\xi_{0}^{2} q_{\infty}^{2}}\right), \quad b=f_{\infty}^{2}\left(4-3 f_{\infty}^{2}\right), \quad c=2 f_{\infty}^{4}\left(f_{\infty}^{2}-1\right)
$$

then, equation (26) gets the form

$$
x^{3}+a x^{2}+b x+c=0 .
$$

It is well-known that the solution of the cube equation has been given by the Kardano-Tartalia's formula. Among three roots of the cubic equation we must take the one which coincides with the result as $f_{\infty}=1$. Thus, for $f_{+}^{2}$ one finds

$$
f_{+}^{2}=\mp \frac{2}{3} \sqrt{a^{2}-3 b} \sin \left(\frac{\pi}{6}-\frac{1}{3} \arctan \sqrt{\frac{4\left(a^{2}-3 b\right)^{3}}{\left(9 b a-27 c-2 a^{3}\right)^{2}}-1}\right)-\frac{a}{3}
$$

here sign $(-)$, at $9 b a-27 c-2 a^{3}>0$ and $(+)$ at $9 b a-27 c-2 a^{3}<0$. The obtained expression (31) is the boundary value of the function $f(\zeta)$ at $\zeta=a / 2$. It allows us to find a constant $C$ which is included in the solution of the Ginzburg-Landau equation. 


\section{The current density in an $S N I N S$ junction}

Now let us consider the equilibrium current states, which can exist in an SNINS junction. As a starting point the common expression for current in the Ginzburg-Landau theory [3,8] has been taken

$$
j(\zeta)=\mathrm{i} \frac{7 \zeta(3)}{16 \pi^{2}} \frac{e n v_{0}}{p_{0} \xi_{0} T_{\mathrm{c}}^{2}}\left(\Delta \frac{d \stackrel{*}{\Delta}}{d \zeta}-\stackrel{*}{\Delta} \frac{d \Delta}{d \zeta}\right) .
$$

We shall calculate the density of the current in the region $\xi_{0} \ll z \ll \xi(T)$, where both the linear integral equation and the Ginzburg-Landau equation are valid. Then one can use the asymptote of the solution of the linear integral equation for $\Delta(z)$ at $|z| \gg \xi_{0}$ which is linear

$$
\begin{array}{ll}
\Delta(\zeta)=\Delta_{+}^{\prime} \zeta+\Delta_{+}, & \zeta \rightarrow+\infty, \\
\Delta(\zeta)=\Delta_{-}^{\prime} \zeta+\Delta_{-}, & \zeta \rightarrow-\infty .
\end{array}
$$
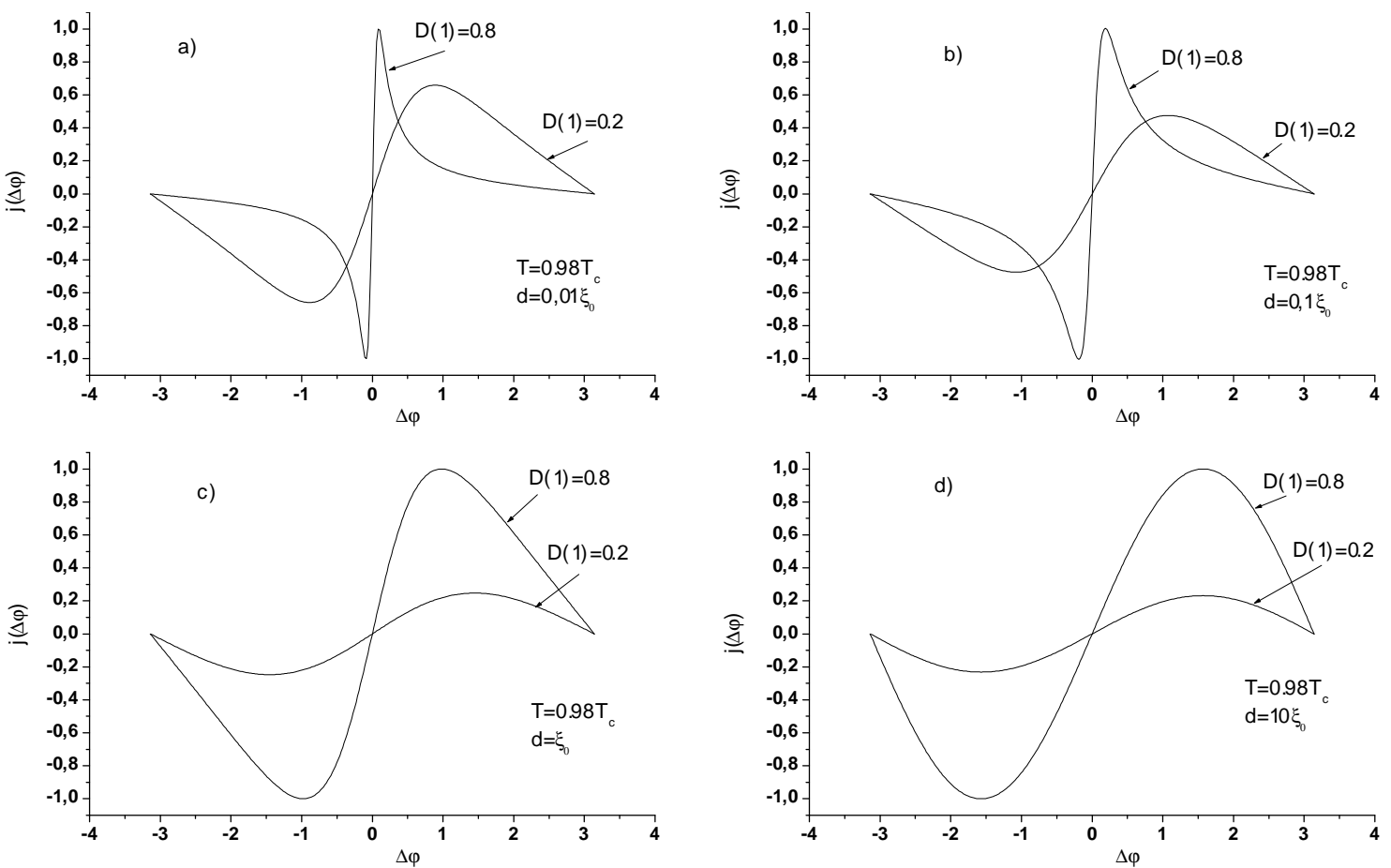

Figure 2. Current-phase relation in $S N I N S$ junction at $T / T_{\mathrm{c}}=0.98$ for different values of the thickness of the normal layer $d$ and for different barrier transparencies $D(1)$. The current density is divided by the maximum value for $D(1)=0.8$.

Let us substitute (33) into (32) and use representation (16). Thus, one gets

$$
j=\frac{1}{2} \frac{e n v_{0}}{p_{0} \xi_{0}}\left(1-\frac{T}{T_{\mathrm{c}}}\right)\left(\frac{1}{q_{2, \infty}}-\frac{1}{q_{1, \infty}}\right) f_{+}^{2} \sin \varphi .
$$

Here the symmetry of the junction is used. It gives $f_{+}=f_{-}, \quad f_{+}^{\prime}=-f_{-}^{\prime}$ (see [9]). Taking into account (31) finally we have

$$
j=\frac{1}{3} \frac{e n v_{0}}{p_{0} \xi_{0}}\left(1-\frac{T}{T_{\mathrm{c}}}\right)\left(\frac{1}{q_{2, \infty}}-\frac{1}{q_{1, \infty}}\right)\left\{\mp \sqrt{a^{2}-3 f_{\infty}^{2}\left(4-3 f_{\infty}^{2}\right)}\right.
$$




$$
\left.\times \sin \left(\frac{\pi}{6}-\frac{1}{3} \operatorname{arctg} \sqrt{\frac{4\left(a^{2}-3 f_{\infty}^{2}\left(4-3 f_{\infty}^{2}\right)\right)^{3}}{\left(9 f_{\infty}^{2}\left(4-3 f_{\infty}^{2}\right) a-54 f_{\infty}^{4}\left(f_{\infty}^{2}-1\right)-2 a^{3}\right)^{2}}-1}\right)-\frac{a}{2}\right\} \sin \varphi
$$

here

$$
a=-2\left[1+\frac{7 \zeta(3)}{12\left(1-T / T_{\mathrm{c}}\right)}\left(\frac{\cos ^{2} \frac{\varphi}{2}}{q_{1, \infty}}+\frac{\sin ^{2} \frac{\varphi}{2}}{q_{2, \infty}}\right)^{2}\right]
$$

From figure 2 it follows that the dependence of the current density on the phase difference between the banks of the junction is strongly nonsinusoidal in Josephson junction SNINS type with the thin normal metal layer at the high barrier transparency and the temperature close to critical.

Both the current-phase and the current-thickness relations $d \gg 1 / p_{0}$ are taken for analysis. The reason is that in the Green's function $G_{\omega_{n}}^{(0)}\left(\vec{r}, \vec{r}^{\prime}\right)$ we ignore the terms, which are the fast oscillation (on the distance order $\left.1 / p_{0}\right)$. In fact we used a quasiclassical approximation.

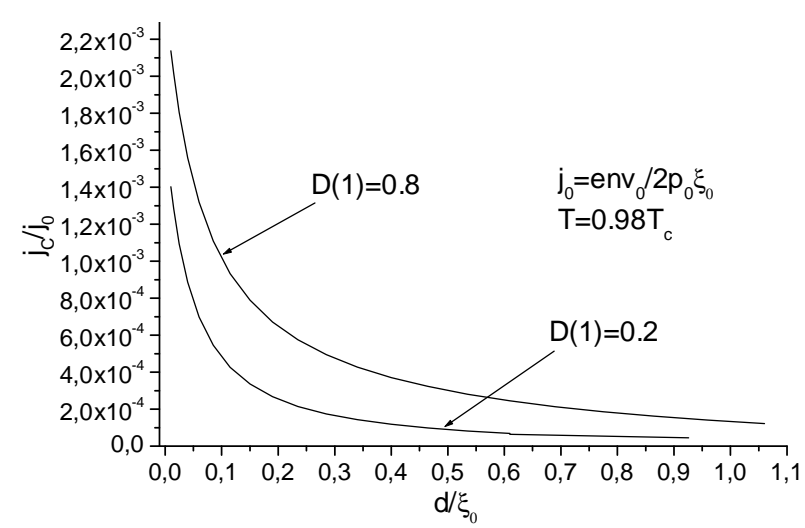

Figure 3. The critical current as a function of the mean normal layer thickness $d$ for different values of the barrier transparency $D(1)$.

\section{Conclusion}

The main results of this study are as follows.

(I) There is a common scheme for building the linear integral equation which describers the behaviour of the order parameter near $N S$ interface.

(II) The boundary condition for the Ginzburg-Landau equation is obtained using the linear integral equation and the method of quasiorthogonality to asymptotics. The boundary condition involves: a barrier transparency, an interlayer thickness, a temperature, a phase difference.

(III) The Ginzburg-Landau equation is solved taking into account the effect of the current on the spatial behaviour of the order parameter. It is shown that the presence of the current in a junction reduces the value of the order parameter in comparison with the currentless case.

(IV) The main result of this work is formula (35) for the current states in a SNINS junction for an arbitrary thickness of the normal layer. Both current-phase and current-thickness relations are shown in figures 2,3 . As a result, one can see that the dependence of the current density on the phase difference between the banks of the junction is strongly nonsinusoidal for the thin $\left(d \ll \xi_{0}\right)$ normal metal layer at a high barrier transparency and at the temperature close to critical. 


\section{References}

1. Svidzinsky A.V., Antsygina T.N, Bratus' E.N., Zh. Eksp. Teor. Fiz., 1971, 61, No. 4, 1612 (in Russian); Sov. Phys. JETP., 1971, 34, 860.

2. Svidzinsky A.V. The theory of Josephson current in the supercoducting junction whith nomal layer. - In: Modern Problems of Statistical Physics. Kiev, 1985, 245-255.

3. Svidzinsky A.V. Space-inhomogeneous Problem of the Superconducting Theory. Moscow, 1982.

4. Golubev L.V., Rakov Y.A., Svidzinsky A.V., Fiz. Mnogochast. Sys., 1985, 7, 40 (in Russian).

5. Akhramovich L.N., Svidzinsky A.V., Fiz. Nizk. Temp., 1988, 14, 815 (in Russian).

6. Svidzynsky A.V., Viligursky O.M., Biruk O.M., Rakutsky A., Journal Physical Studies, 1999, 3, No. 3, 359 .

7. Akhramovich L.N., Rakov Y.A., Svidzinsky A.V., Teor. Mat. Fiz., 1988, 77, 450 (in Russian).

8. Svidzinsky A.V. The Microscopic Theory of Superconductivity. Part 1. Lutsk, 2001.

9. Svidzinsky A.V. The Microscopic Theory of Superconductivity. Part 2. Lutsk, 2003.

10. Svidzinsky A.V. Mathematical Methods of The Theoretical Physics. Kyiv, 1998.

11. Svidzinsky A.V., Sakhnyuk V.E., Condens. Matter Phys., 2000, 3, 683.

12. Sakhnyuk V.E., Svidzinsky A.V., Condens. Matter Phys., 2003, 6, 159.

\section{До теорії струмових станів в $S N I N S$ контакті}

\section{В.Є.Сахнюк, А.В.Свідзинський}

Волинський державний університет імені Лесі Українки, кафедра теоретичної і математичної фізики,

проспект Волі,13, Луцьк 43000, Україна

Отримано 3 серпня 2005 р., в остаточному вигляді 8 листопада 2005 р.

Розглянуто поведінку параметра впорядкування поблизу границі розділу нормальної та надпровідної області в $S N I N S$-контакті. З цією метою одержано і досліджено лінійне інтегральне рівняння, яке $є$ справедливим поблизу границі. Розв'язано рівняння Гінзбурга-Ландау з врахуванням впливу струму на просторову поведінку параметра впорядкування. Для рівняння Гінзбурга-Ландау, використовуючи метод квазіортогональності до асимптотики, отримано граничну умову. Виконано розрахунок для густини струму, який може протікати через $S N I N S$-контакт. При цьому товщина нормального прошарку вважається довільною, а температура близькою до критичної.

Ключові слова: надпров ідний контакт, $N S$-границя, параметр впорядкування, лінійне інтегральне рівняння, рівняння Гінзбурга-Ландау, асимптотика

PACS: $74.50 .+r$ 
